\title{
A method for 3 dimensional structural and compositional imaging of nano-materials
}

\author{
T. Yaguchi*, Y.Kuroda*, M.Konno*, T. Kamino*, K.Kaji** and M.Watanabe*** \\ * Hitachi High-Technologies Corp., 11-1 Ishikawa-cho, Hitachinaka, Ibaraki, 312-0057 Japan \\ **Hitachi High-Technologies Corp., 882 Ichige, Hitachinaka, Ibaraki, 312-8504 Japan \\ ***Dept. of Materials Science \& Engineering, Lehigh University, Bethlehem, PA, 18015 USA
}

In the fields of electronic devices and nano-materials, demands for three dimensional (3D) imaging are increasing rapidly. Recently, we have developed a method for multi-directional observation of a specific site at atomic level resolution [1], [2]. In this method, the specimen is prepared using the FB-2100 focused ion beam (FIB) system equipped with a FIB micro-sampling system [3], [4] and a FIB-scanning transmission electron microscopy (STEM) / transmission electron microscopy (TEM) compatible specimen rotation holder. This technique allows milling of the specimen and STEM/TEM observation to be carried out alternately so that $3 \mathrm{D}$ imaging of a specific site can be performed at the optimized specimen thickness. The specimen is FIB milled to a pillar shape and mounted on the tip of a needle stub which is held in the rotation mechanism of the specimen holder. The specimen holder allows $360^{\circ}$ rotation and $\pm 20^{\circ}$ tilting of a pillar shaped specimen in the narrow-gapped high resolution pole-piece of a TEM or STEM. With this technique several sets of X-ray maps can be obtained using different tilt and rotation series, and 3D elemental distributions can be extracted with very high precision [5]. High resolution STEM/TEM images such as crystal lattice fringes can be observed from various directions. Since the reduction of FIB damage is one of the important issues for high resolution image observation, a low energy Ar ion milling technique was used in the final stage of a sample preparation. A newly developed GENTLE MILL HI low energy Ar ion milling system operated at $200 \mathrm{~V}$ was used for the reduction of the FIB damage. The Ar ion milling system has been modified to permit the direct insertion of the FIB-STEM/TEM compatible specimen rotation holder. Figure 1 shows a TEM image of the Si (110) plane (a) and the corresponding electron diffraction pattern (b) observed after the specimen was FIB milled at 40kV. A TEM image and the corresponding electron diffraction pattern taken after the specimen was Ar ion milled at $200 \mathrm{~V}$ are shown in Fig.1c and Fig.1d, respectively. The microscope used for the high resolution TEM observation was a Hitachi H-9500 300kV high resolution TEM. After the Ar ion milling, the thickness of the amorphous layer was reduced from $28 \mathrm{~nm}$ to $1.2 \mathrm{~nm}$ and the contrast of the halo-ring in the diffraction pattern was weakened. Figure 2 shows high resolution TEM images (a-c) of a pillar shaped Si single crystal specimen and corresponding diffraction pattern (d-f). The crystal lattice fringes of the Si (110), (100), (1-10) planes are clearly observed in the pillar shaped specimen of $120 \mathrm{~nm} \times 150 \mathrm{~nm}$. In addition, the low energy Ar ion milling improves quality of both high resolution TEM images and 3 dimensional elemental maps. 


\section{References}

[1] T. Yaguchi et al., Proc. Microsc. Microanal. 9 (Suppl .2) (2003) 118-119.

[2] T. Yaguchi et al.,Proc. Microsc. Microanal. 10 (Suppl .2) (2004) 1164-1165.

[3] T.Ohnishi, et al. : Proc. 25 th Int.Symp. for Testing and Failure Analysis (1999) 449-453.

[4] US patent USP5270552.

[5] T. Yaguchi et al., Proc. Microsc. Microanal. 11 (Suppl .2) (2005) 630-631.
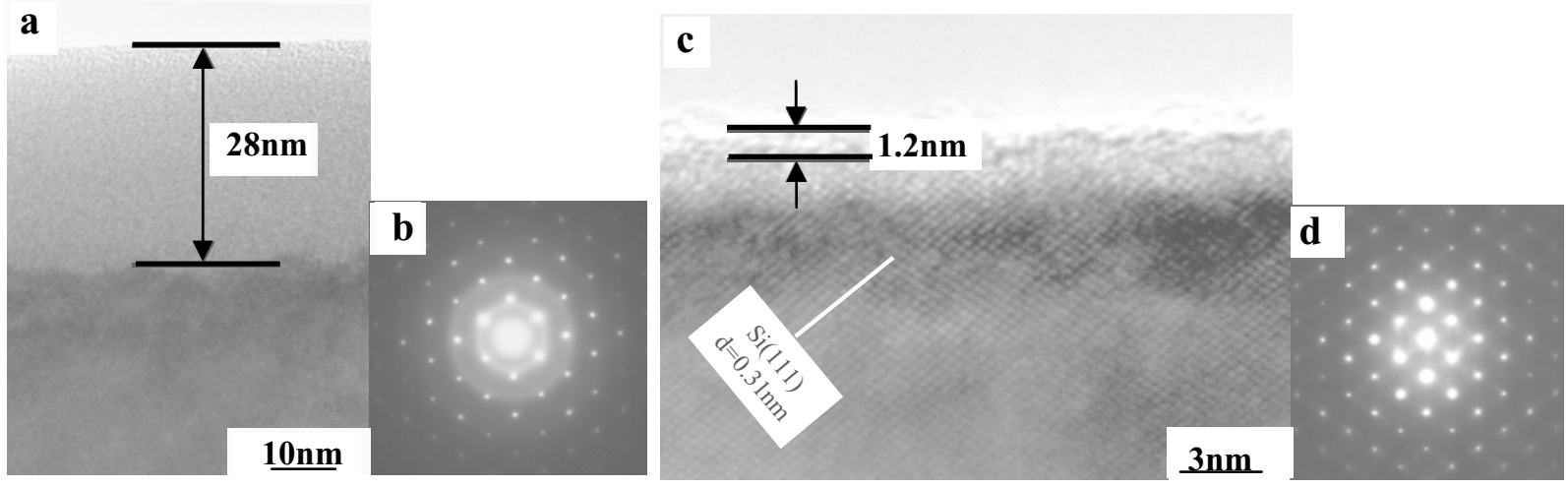

Fig.1 TEM image of the Si (110) plane (a) and the corresponding electron diffraction pattern (b) observed after the specimen was FIB milled at 40kV. A TEM image (c) and the corresponding electron diffraction pattern (d) taken after Ar ion milling the sample at $200 \mathrm{~V}$.

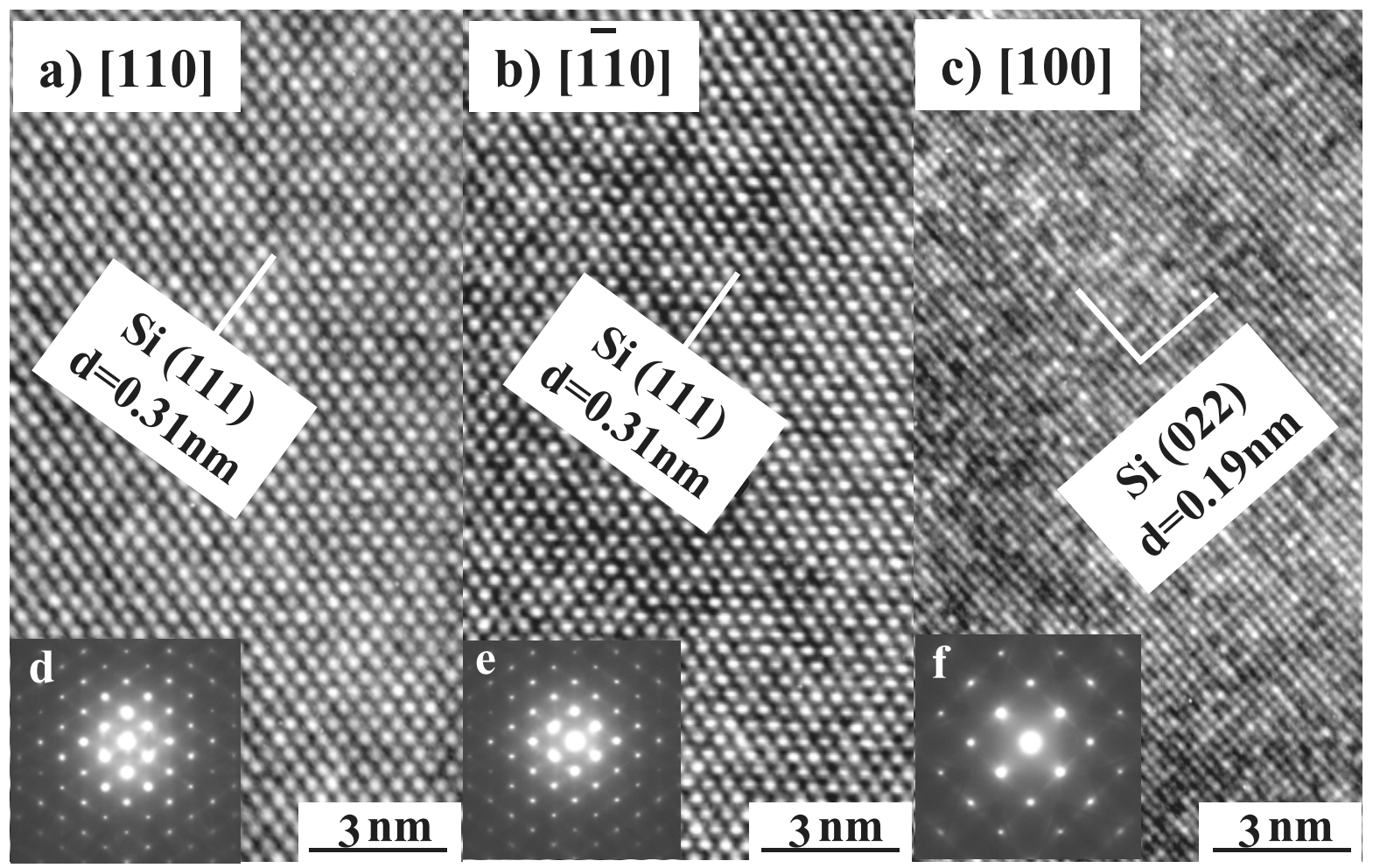

Fig.2 High resolution TEM images(a-c) and electron diffraction patterns (d-f) observed from three directions.Specimen : Si-device, Accelerating voltage : $300 \mathrm{kV}$ 